\title{
Java Language for Numerical Control Simulation System Research
}

\author{
Li Hongmei, Li Lei, and Zhou Huiqiang
}

\begin{abstract}
Java language is very good in a variety of development platforms to develop all kinds of application software for its simple and efficient, widely used. The programming language owning platform independent is adopted to solve the image flicker, sound loading and other issues by the threading technology, multimedia technology, graphics and point by point comparison techniques.Dynamic real-time simulation process simulation is implemented and a two-dimensional network of CNC machining simulation system is developed while Java Applet application is as a carrier. The simulation results show that the system has a friendly interface and fast calculation speed and platform portability has a certain practicality and application value.
\end{abstract}

Index Terms-Network Numerical Control (NC), java language, by-point comparison interpolation, simulation.

\section{INTRODUCTION}

With advanced manufacturing and computer network technology, especially the rapid development of numerical control system of the network, integration has become an inevitable trend [1]. Network CNC System (Network Numerical Control) as a communications and resources (such as processing procedures, machine tools, inspection and monitoring equipment, etc.) is shared as a means to the workshop as well as manufacturing equipment within the enterprise as the goal of the organic integration, support for ISO-OSI networking specification autonomous CNC system [2].

CNC machining process simulation is the primary key of virtual manufacturing technology. It is a computer software technology to simulate the processing environment, the tool path and the material removal process, which simulate the machining process [3]. It is a powerful tool for the reliability of the NC program verification, to prevent the occurrence of interference and collision and forecast process provides [4].

Dynamic simulation of $\mathrm{CNC}$ machining which has important application value is necessary for high-end CNC system functions because it is technical complexity, difficulty [5]. Java is a cross-platform, object-oriented language. It is simple, portable, platform-independent with strong security, and provides distribution, dynamic support. Meanwhile, Java is also rich class library, so that programmers can build their own systems easily. Therefore, a platform independent Java language is used to design and development of the network $\mathrm{NC}$ machining simulation system for $\mathrm{NC}$ machining process

Manuscript received September 4, 2013; revised November 15, 2013.

The authors are with Zheng Zhou Technical College, Zhengzhou 450121, China (e-mail: lhmzjy2003@163.com, lilei20031010@126.com, zhouhuiqiang1973@163.com). simulation.

\section{JAVA INTRODUCTION}

Nowadays, there are a wide variety of programming languages. Facing increasingly demanding customer needs, we need more powerful, more flexible language editing language. Java language in such a context emerged. Java by Sun Microsystems, Inc. in May 1995 launch of the Java programming language (hereinafter referred to as the Java language) and the Java platform in general.

Java implementation of the Hot Java browser (Java applet support) shows the charm of Java: cross-platform, dynamic Web, Internet computing. Java has been widely accepted and promoted the rapid development of the Web since then. Popular browsers now support Java applet. On the other hand, Java technology has also been updated.

Java platform is composited of the Java Virtual Machine (Java Virtual Machine, JVM) and Java application programming (Application Programming Interface, API).Java application programming interface for the Java application provides an operating system-independent standard interface, can be divided into basic parts and extensions. In the hardware or operating system platform after installing a Java platform, Java applications can be run. Java platform is now already embedded in almost all operating systems.

So that Java programs can compile only once, you can run in a variety of systems. Java application programming interface has been developed from version $1.1 x$ to version 1.2. The most commonly used Java platform is based on Java1.4, the most recent version of Java 1.7.

\section{FIVE CHARACTERISTICS OF JAVA}

\section{A. Simple and Flexible}

$\mathrm{C}$ language, $\mathrm{C}++$, Java language is common programming languages. Where $\mathrm{C}$ language syntax is more complex and difficult to understand, $\mathrm{C}++$ application programming technically demanding, difficult to understand, easy to confuse .Therefore, programmers often make it difficult to learn and accept. In contrast, Java language is relatively simple, not too high syntax and programming technical requirements and Java language features in the integrated program also has the incomparable advantage of other programming languages, it is often the first choice as a WEB development, its development is relatively mature.

\section{B. Object-Oriented Features}

Unlike $\mathrm{C}++$, java language can only function as inheritance, 
but also includes a variety of classes and other attributes. Java language can be regarded as an object-oriented, dynamic mechanism for the overall operation of program design. Object-oriented programming language Java has four basic characteristics: encapsulation, polymorphism, inheritance and dynamic binding.

\section{Better Reliability and Network Security}

Java language can use built-in mechanisms to prevent other malicious code attacks, and use built-in safety mechanism preventing the network to download the package, class analysis to achieve the program runs. The Java language is often used for common network environments because of its security.

\section{Java Language Can Operate in Parallel}

In the Java operating environment, Thread class and Runnable interface objects through a unique way of preparation and operation of programs related to the library to create a special kind of object - thread. Based on this preparation methods and operating procedures, Java language has higher efficiency, multithreading can work together or in parallel and independently of each other.

\section{E. Dynamic}

We had to develop such a language so that real-time data can be manipulated through language in a dynamic environment for data manipulation. Java language came into being while it can affect the operation of the program under the premise of the editing operations through dynamic classes and packages will be transferred to the operating system environment. It can also live on the packages and classes for calling and downloading.

\section{DEVELOPMENT PlATFORM}

\section{A. Operating Systems}

Windows XP operating system is selected for software developers and run, but the Java language with platform independence is developed because you can either run under the operating system.

\section{B. Development Tools}

Borland's integrated development tools Jbuilder 9.0 which supports visual programming and object-oriented technology is selected. Jbuilder 9.0 developed by the Borland Company enhances the WEB development technologies. Who can make the different regions together, while research and development with a project to solve the team running, causing a lot of unnecessary human and financial loss. The development of this environment is important in order to run the latest version of Java, to get better and more functional results. Customer-related operations by the builder, you can achieve the following aspects: First, you can get the required database tables Java classes; Second, the development of e-commerce platform to achieve a variety; Third, combining the use of Java language development dynamic WEB, graphics mode window and threads to achieve parallel operation.

\section{The IMPLEMENTATION OF Key TECHNOLOGIES}

\section{A. The Implementation of Real-Time Simulation}

Simulation of real-time simulation of the system operation to meet the requirements of a certain time constraints, the control simulation time and machine time to meet specified constraint. In order to achieve real-time processing of the simulation, the system uses multi-threading. Java multi-threading support is a technology major feature, use it to achieve concurrent execution of multiple tasks to improve the interpolation operation speed, effectively control the simulation time, to solve the real-time process simulation problems.

\section{B. Dynamic Simulation Realization}

The most commonly used in animation is to load multiple images are sequentially displayed according to a certain order, the formation of the animation. This paper uses the Java language to achieve self-drawn graphics animation.

Call sleep () method

In order to continuously display the time interval between the images remain stable, called sleep () method implementation delays. In the method, you can set the delay time

The process of solving animation flickering problem

Animation process, is the process of constantly refresh the screen, but will produce annoying flicker. To solve the flicker problem, this paper is to show the animation process, first define what makes animated images the same size, the same location, and then use the default paint mode, so that the image shows a continuous process, the image is completely behind the front cover images, thereby unnecessarily clear the screen, and finally by removing the update () method of the statement is clear the screen, eliminating the animation flicker.

\section{NC INTERPOLATION (INTERPOLATION)}

The so-called interpolation (Interpolation) is based on a given feed rate and linearity requirements given contour, the contour between known points to determine some intermediate point algorithms. The system uses a point by point comparison method CNC interpolation.

\section{A. By-Point Comparison Linear Interpolation}

\section{1) Deviation functions}

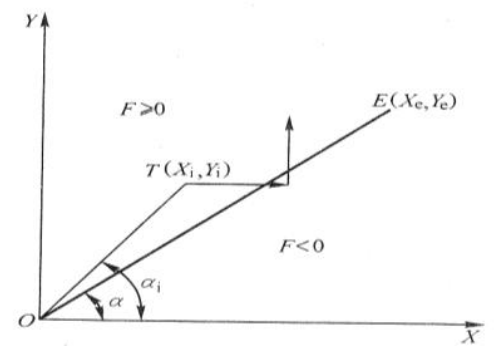

Fig. 1. Schematic linear interpolation.

The first quadrant linear interpolation principle is shown in Fig. 1. The programming line to be processed is given when the start and end point of the line terms of origin, the end point coordinates of $E\left(X_{e}, Y_{e}\right)$, point $P\left(X_{i}, Y_{i}\right)$ represents the 
position of the tool.

If the point $\mathrm{P}$ is exactly on the line, the following equation is established.

$$
Y_{i} / X_{i}=Y_{e} / X_{e} \text {, namely } X_{i} Y_{e}-Y_{i} X_{e}=0
$$

If the point $\mathrm{P}$ on the line just above the line, then

$$
Y_{i} / X_{i}>Y_{e} / X_{e} \text {, namely } X_{i} Y_{e}-Y_{i} X_{e}>0
$$

If the point $\mathrm{P}$ is just below the line, then

$$
Y_{i} / X_{i}<Y_{e} / X_{e} \text {, namely } X_{i} Y_{e}-Y_{i} X_{e}<0
$$

From the above, we may take the function $F=X_{i} Y_{e}-Y_{i} X_{e}$ as the deviation of a discriminant function which is called deviation function. The function $F\left(X_{i}, Y_{i}\right)$ can determine the value of the current point and the relative position of the line, i.e.

When $F\left(X_{i}, Y_{i}\right)=0$, the tool in a straight line;

When $F\left(X_{i}, Y_{i}\right)>0$ when the tool is above the line;

When $F\left(X_{i}, Y_{i}\right)<0$, the tool is below the line.

\section{2) Deviation function recursive calculation}

Deviation function using recursive type, that is calculated from the previous point a little. If $F_{i} \geq 0$, a step along the $+X$ direction, the new coordinates and deviation is shown:

$$
\begin{gathered}
X_{i+1}=X_{i}+1, Y_{i+1}=Y_{i} \\
F_{i+1}=X_{e} Y_{i}-\left(X_{i}+1\right) Y_{e}=X_{e} Y_{i}-X_{i} Y_{e}-Y_{e}=F_{i}-Y_{e}
\end{gathered}
$$

If $F i \leq 0$, a step along the $+Y$ direction, the new coordinates and deviation is shown:

$$
\begin{gathered}
X_{i+1}=X_{i}, Y_{i+1}=Y_{i}+1 \\
F_{i+1}=X_{e}\left(Y_{i}+1\right)-X_{i} Y_{e}=X_{e}+X_{e} Y_{i}-X_{i} Y_{e}=F_{i}+X_{e}
\end{gathered}
$$

\section{3) End discrimination}

Every step of the tool feed, should be carried out once the end discrimination, have already reached the end, interpolation operation is stopped, and issued a new block down or converted signal, otherwise, proceed interpolation cycle. By-point comparison interpolation flowchart in Fig. 2.

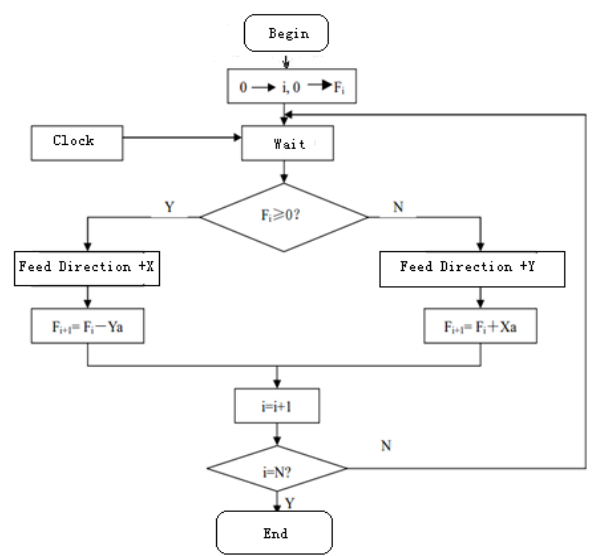

Fig. 2. Flowchart interpolation point by point comparison.

\section{B. Circular Interpolation Point by Point Comparison}

\section{1) Deviation function construction}

Shown in Fig. 3, assuming that the part contour to be machined first quadrant inverse arc AE, the tool at point $T\left(X_{i}, Y_{i}\right)$, with the center of $O(0,0)$ of radius $R$. When the point $T\left(X_{i}, Y_{i}\right)$ just landed on $A E$ arc, then the following holds:

$$
X_{i}^{2}+Y_{i}^{2}=X_{e}^{2}+Y_{e}^{2}=R^{2}
$$

When the point $T\left(X_{i}, Y_{i}\right)$ falls outside the arc AE, then the following equation is:

$$
X_{i}^{2}+Y_{i}^{2}>X_{e}^{2}+Y_{e}^{2}=R^{2}
$$

When the point $T(X i, Y i)$ falls inside the $\operatorname{arc} A E$, then the following equation is:

$$
X_{i}^{2}+Y_{i}^{2}<X_{e}^{2}+Y_{e}^{2}=R^{2}
$$

According to the above, we take circular interpolation function expression for the deviation:

$$
F=X_{i}^{2}+Y_{i}^{2}-R^{2}
$$

When $F \geq 0$, the fixed point outside the circle or on the circle, according to the principles established in the feed direction, the tool along the- $X$ direction feed step; when $F<0$, the point inside the arc, the tool along the $+Y$ direction feed step.

\section{2) A new function to calculate the deviation}

If $F_{i} \geq 0$, the tool along the- $X$-axis direction feed step to reach the new coordinate position

$$
X_{i+1}=X_{i}-1, Y_{i+1}=Y_{i}
$$

Therefore, the new deviation function

$$
\begin{gathered}
F_{i+1}=X_{i+1}{ }^{2}+Y_{i+1}{ }^{2}-R^{2}=\left(X_{i}-1\right)^{2}+Y_{i}^{2}-R^{2} \\
F_{i+1}=F_{i}-2 X_{i}+1
\end{gathered}
$$

Similarly, if $F i<0$, the tool along the $+Y$-axis direction feed step to reach the new coordinate position:

$$
X_{i+1}=X_{i}, Y_{i+1}=Y_{i}+1, \quad F_{i+1}=F_{i}+2 Y_{i}+1
$$

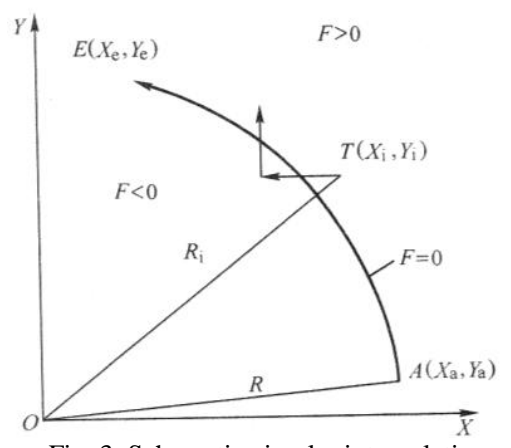

Fig. 3. Schematic circular interpolations.

\section{3) End discrimination}

Every step of the tool feed, should be carried out once the end discrimination, have already reached the end, 
interpolation operation is stopped, and issued a new block down or converted signal, otherwise, proceed interpolation cycle. End discrimination is calculated as:

$$
\sum=\left|X_{e}-X_{a}\right|+\left|Y_{e}-Y_{a}\right|
$$

Circular interpolation point by point comparison flowchart is shown in Fig. 4.

By Shanghai Machine Tool Factory models for CK6150 $\mathrm{CNC}$ lathe Fig. 5, for instance validation. Use and development of the simulation system for the first quadrant linear and circular machining process simulation, the simulation results shown in Fig. 6, Fig. 7.

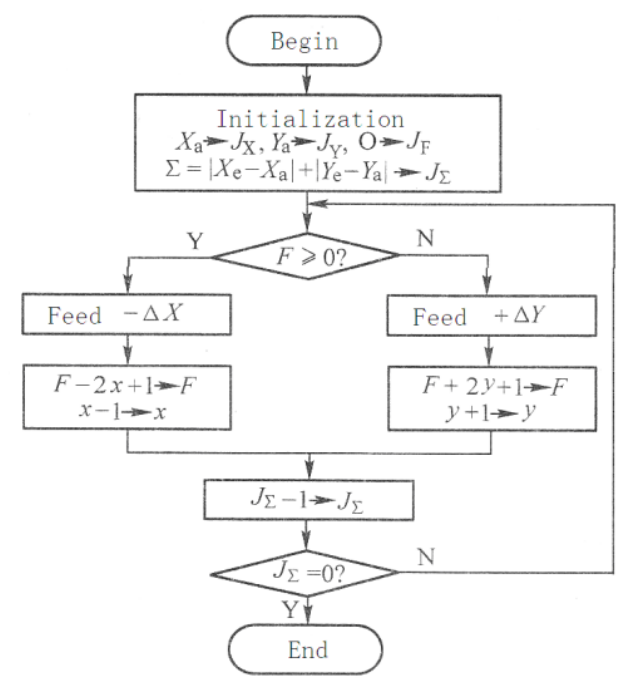

Fig. 4. By-point comparison method flowchart first quadrant circular interpolation.

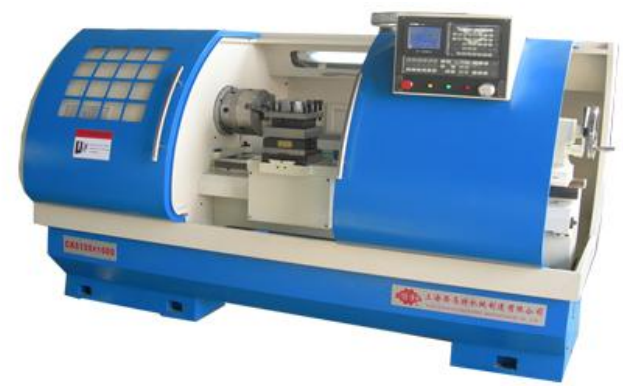

Fig. 5. CK6150 CNC lathe.

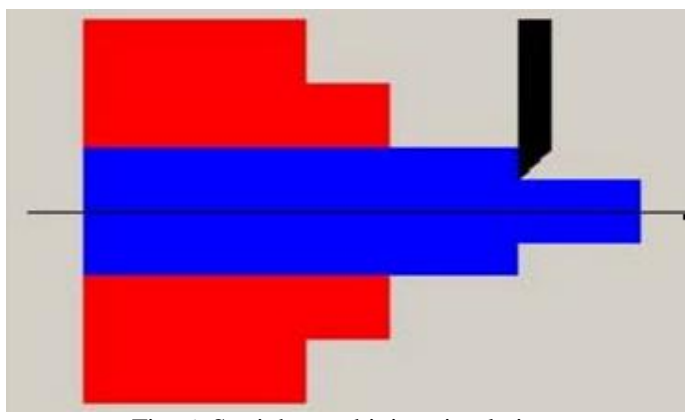

Fig. 6. Straight machining simulation.

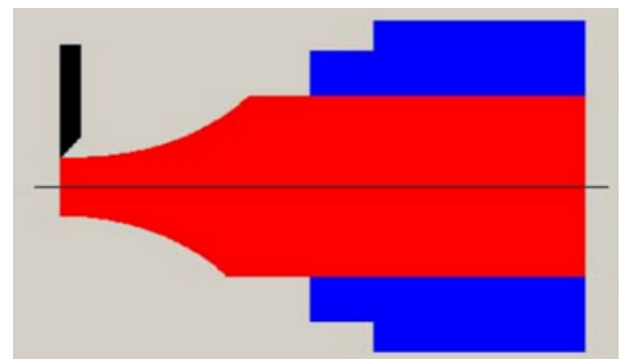

Fig. 7. Arc machining simulation.

\section{CONCLUSION}

Platform independent Java programming language to Java Applet application as the carrier, to solve the simulation process image flicker, sound, load and other issues, to achieve a real-time simulation of dynamic simulation, the completion of the network of two $\mathrm{NC}$ dimensional machining simulation system development. And CK6150 CNC lathe, a straight line, arc, test verification process, the simulation results show that the system has a friendly interface and fast calculation speed and platform portability, has a certain practicality and application value.

\section{REFERENCES}

[1] Z. J. Sun and Y. L. Huang, CNC Machining Technology, Anhui Science and Technology Press, pp. 23-24, 2009.

[2] J. Wang, CNC Programming and Machining Technology, Tsinghua University Press, pp. 3-5, 2009.

[3] J. Zhang and W. X. Wang, "Java-based network NC machining simulation system development," Southwest University for Nationalities, Natural Science Edition, vol. 5, 2009.

[4] X. W. Wang, "Java object-oriented programming language and its application," Computers, vol. 12, 1996.

[5] Goofy, "WEB applications using the Java language to develop software knowledge explore," Electronic World, vol. 5, 2012.

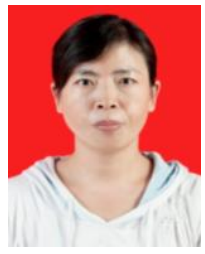

Li Hongmei was born in 1972 in Qi County of Henan. She graduated from Henan University. She is currently a lecturer at Zhengzhou Technical College. Her area of research interests are in computer applications.

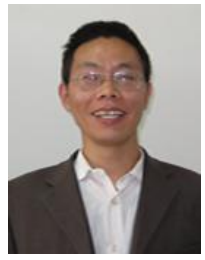

Li Lei was born in 1972. He received his Ph.D. from Beijing Institute of Technology in 2011. At present he has worked at Zheng Zhou technical college. His area of research interests are in explosion mechanics.

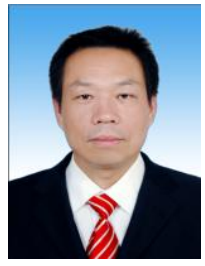

Zhou Huiqiang was born in 1973 in Yichuan, Henan He graduated from Zhengzhou University and now is a lecturer at Zhengzhou Technical College. His area of research interests are in computer simulation technology. 